\title{
Analyzing the effect of nozzle diameter in fused deposition modeling for extruding polylactic acid using open source 3D printing
}

\begin{abstract}
Fused deposition modeling (FDM) is one of the Rapid Prototyping (RP) technologies. The 3D Printer has been widely used in the fabrication of 3D products. One of the main issues has been to obtain a high quality for the finished parts. The present study focuses on the effect of nozzle diameter in terms of pressure drop, geometrical error as well as extrusion time. While using polylactic acid (PLA) as a material, the research was conducted using Finite Element Analysis (FEA) by manipulating the nozzle diameter, and the pressure drop along the liquefier was observed. The geometrical error and printing time were also calculated by using different nozzle diameters. Analysis shows that the diameter of the nozzle significantly affects the pressure drop along the liquefier which influences the consistency of the road width thus affecting the quality of the product's finish. The vital aspect is minimizing the pressure drop to be as low as possible, which will lead to a good quality final product. The results from the analysis demonstrate that a $0.2 \mathrm{~mm}$ nozzle diameter contributes the highest pressure drop, which is not within the optimum range. In this study, by considering several factors including pressure drop, geometrical error and printing time, a $0.3 \mathrm{~mm}$ nozzle diameter has been suggested as being in the optimum range for extruding PLA material using open-source 3D printing. The implication of this result is valuable for a better understanding of the melt flow behavior of the PLA material and for choosing the optimum nozzle diameter for $3 \mathrm{D}$ printing.
\end{abstract}

Keyword: Nozzle diameter; Pressure drop; Fused deposition modeling; Open source 3D printing 